\title{
A New Subclass of Meromorphic Close-to-Convex Functions
}

\author{
Amit Soni ${ }^{1}$ and Shashi Kant ${ }^{2}$ \\ ${ }^{1}$ Department of Mathematics, Government Engineering College, Bikaner 334001, India \\ ${ }^{2}$ Department of Mathematics, Government Dungar College, Bikaner 334001, India
}

Correspondence should be addressed to Amit Soni, aamittsonni@yahoo.com

Received 31 August 2012; Revised 13 November 2012; Accepted 13 November 2012

Academic Editor: Alfonso Montes-Rodriguez

Copyright (C) 2013 A. Soni and S. Kant. This is an open access article distributed under the Creative Commons Attribution License, which permits unrestricted use, distribution, and reproduction in any medium, provided the original work is properly cited.

A new subclass $M K(t, A, B)$ of meromorphic close-to-convex functions, defined by means of subordination, is investigated. Some results such as inclusion relationship, coefficient inequality, convolution property, and distortion property for this class are derived. The results obtained here are extension of earlier known work.

\section{Introduction}

Let $\sum$ denote the class of functions $f$ of the form

$$
f(z)=\frac{1}{z}+\sum_{n=1}^{\infty} a_{n} z^{n}
$$

which are analytic in the punctured open unit disk $\mathbb{D}=\{z$ : $z \in \mathbb{C}: 0<|z|<1\}$. For any two analytic function $f$ and $g$ in $\mathbb{U}=\mathbb{D} \bigcup\{0\}$, we say that $f$ is subordinate to $g$ in $\mathbb{U}$, written as $f(z)<g(z)$, if there exists a Schwarz function $w(z)$ such that $f(z)=g(w(z))$, for $z \in \mathbb{U}$. In particular, if $g$ is univalent in $\mathbb{U}$, then $f$ is subordinate to $g$, if and only if $f(0)=g(0)$ and $f(\mathbb{U}) \subset g(\mathbb{U})$. If $f \in \sum$ is given by (1) and $g \in \sum$ is given by

$$
g(z)=\frac{1}{z}+\sum_{n=1}^{\infty} b_{n} z^{n},
$$

then the Hadamard product (or convolution) of the function $f$ and $g$ is defined by

$$
(f * g)(z)=\frac{1}{z}+\sum_{n=1}^{\infty} a_{n} b_{n} z^{n}=(g * f)(z) .
$$

A function $f \in \sum$ is said to be in the class $M S^{*}(\alpha)$ of meromorphic starlike functions of order $\alpha$ if it satisfies the inequality

$$
R\left(\frac{z f^{\prime}(z)}{f(z)}\right)<-\alpha, \quad(z \in \mathbb{U}, 0 \leq \alpha<1) .
$$

Moreover, a function $f \in \sum$ is said to be in the class $M C$ of meromorphic close to convex function if it satisfies the condition

$$
R\left(\frac{z f^{\prime}(z)}{g(z)}\right)<0 \quad\left(z \in \mathbb{U}, g \in M S^{*}(0)=M S^{*}\right) .
$$

Recently, Wang et al. [1] introduced and discussed the class $M K$ of meromorphic functions $f(z) \in \sum$ which satisfies the inequality

$$
R\left(\frac{f^{\prime}(z)}{g(z) g(-z)}\right)>0 \quad(z \in \mathbb{U}),
$$

where $g(z) \in M S^{*}(1 / 2)$.

The class $M K$ is very close to the interesting subclass $K_{s}$ of close-to-convex function for analytic function introduced and studied by Gao and Zhou [2]. Many classes related to the class $K_{s}$ have been further studied by some authors. Especially, Wang et al. [3, 4], Kowalczyk and Leś-Bomba [5], Xu et al. [6], Şeker [7], and Cho et al. [8] introduced generalization of $K_{s}$ and obtained some properties for analytic functions in each class.

More recently, by means of subordination, Sim and Kwon [9] discussed a subclass $\sum(A, B)$ of the class $M K$. A function $f(z) \in \sum$ is said be in the class $\sum(A, B)$ if it satisfies the following subordination relation:

$$
\frac{f^{\prime}(z)}{g(z) g(-z)} \prec \frac{1+A z}{1+B z},
$$


where $-1 \leq B<A \leq 1$ and $g(z) \in M S^{*}(1 / 2)$. Here, the assumption that $g(z)$ is meromorphic starlike function of order $1 / 2$ makes the function $-z g(z) g(-z)$ meromorphic starlike. So, instead of $-z g(z) g(-z)$ in (6) and (7), we can consider $\operatorname{tg}(z) g(t z), 0<|t| \leq 1$, because if $g(z) \in M S^{*}(1 / 2)$, then $\operatorname{tg}(z) g(t z)$ is also a meromorphic starlike function, which motivates us to define a new subclass $M K(t, A, B)$ of meromorphic close-to-convex functions as follows.

Definition 1. A function $f \in \sum$ is said to be in the class $M K(t, A, B)$ if there exists $g \in M S^{*}(1 / 2)$, such that

$$
\frac{-f^{\prime}(z)}{\operatorname{tg}(z) g(t z)} \prec \frac{1+A z}{1+B z},
$$

$$
(z \in \mathbb{U}, 0<|t| \leq 1,-1 \leq B<A \leq 1) .
$$

The class $M K(t, A, B)$ provides a generalization of the classes given by Sim and Kwon [9] and Wang et al. [1]. The transformation $(1+A z) /(1+B z)$ involved in the class $M K(t, A, B)$ is analytic and convex univalent in $\mathbb{U}$. Moreover,

$$
\begin{aligned}
0 & \leq \frac{1-A}{1-B}<R\left(\frac{1+A z}{1+B z}\right) \\
& <\frac{1+A}{1+B}, \quad(-1<B<A \leq 1 ; z \in \mathbb{U}) .
\end{aligned}
$$

In this paper, we aim at proving results such as inclusion relationship, coefficient bounds, and distortion theorem for the class $M K(t, A, B)$.

\section{Properties of Meromorphic Starlike Functions}

In beginning, we prove the following result of meromorphic starlike functions.

Theorem 2. Suppose that $\phi \in M S^{*}\left(\alpha_{1}\right)$ and $\psi \in M S^{*}\left(\alpha_{2}\right)$ with $0 \leq \alpha_{1}+\alpha_{2}-1<1$. Then,

$$
\begin{array}{r}
z t_{1} t_{2} \phi\left(t_{1} z\right) \psi\left(t_{2} z\right) \in M S^{*}\left(\alpha_{1}+\alpha_{2}-1\right), \\
\text { where } 0<\left|t_{1}\right|,\left|t_{2}\right| \leq 1 .
\end{array}
$$

Proof. Let $\phi_{i} \in M S^{*}\left(\alpha_{1}\right)$ and $\psi \in M S^{*}\left(\alpha_{2}\right)$. By definition, we know that

$$
\begin{aligned}
& R\left(\frac{t_{1} z \phi^{\prime}\left(t_{1} z\right)}{\phi\left(t_{1} z\right)}\right)<-\alpha_{1}, \\
& R\left(\frac{t_{2} z \psi^{\prime}\left(t_{2} z\right)}{\psi\left(t_{2} z\right)}\right)<-\alpha_{2} .
\end{aligned}
$$

Next, we suppose that

$$
h(z)=z t_{1} t_{2} \phi\left(t_{1} z\right) \psi\left(t_{2} z\right) .
$$

Then, we easily find that

$$
\frac{z h^{\prime}(z)}{h(z)}=1+\frac{t_{1} z \phi^{\prime}\left(t_{1} z\right)}{\phi\left(t_{1} z\right)}+\frac{t_{2} z \psi^{\prime}\left(t_{2} z\right)}{\psi\left(t_{2} z\right)} .
$$

It follows that

$$
R\left(\frac{z h^{\prime}(z)}{h(z)}\right)<-\left(\alpha_{1}+\alpha_{2}-1\right) .
$$

By noting that $0 \leq\left(\alpha_{1}+\alpha_{2}-1\right)<1$, which implies that $h(z) \in M S^{*}\left(\alpha_{1}+\alpha_{2}-1\right)$, it completes the proof of Theorem 2 .

For $\alpha_{1}=\alpha_{2}=1 / 2$ and $t_{1}=1$, Theorem 2 gives the following corollary.

Corollary 3. If $g(z) \in M S^{*}(1 / 2)$ and $0<|t| \leq 1$, then $t z g(z) g(t z) \in M S^{*}$.

Lemma 4 (see [10]). Let $-1 \leq B_{2} \leq B_{1}<A_{1} \leq A_{2} \leq 1$. Then,

$$
\frac{1+A_{1} z}{1+B_{1} z} \prec \frac{1+A_{2} z}{1+B_{2} z} .
$$

Theorem 5. Let $-1 \leq B_{2} \leq B_{1}<A_{1} \leq A_{2} \leq 1$. Then,

$$
M K\left(t, A_{1}, B_{1}\right) \subset M K\left(t, A_{2}, B_{2}\right) .
$$

Proof. Suppose that $f \in M K\left(t, A_{1}, B_{1}\right)$. Then,

$$
\frac{-f^{\prime}(z)}{\operatorname{tg}(z) g(t z)} \prec \frac{1+A_{1} z}{1+B_{1} z} \text {. }
$$

Since $-1 \leq B_{2} \leq B_{1}<A_{1} \leq A_{2} \leq 1$, by Lemma 4 , we have

$$
\frac{-f^{\prime}(z)}{\operatorname{tg}(z) g(t z)} \prec \frac{1+A_{1} z}{1+B_{1} z} \prec \frac{1+A_{2} z}{1+B_{2} z} \text {. }
$$

It implies that $f(z) \in M K\left(t, A_{2}, B_{2}\right)$, which completes the proof.

\section{Coefficient Estimates}

In this section,we obtain the coefficient estimates of functions belonging to the class $M K(t, A, B)$. For this, we require the following Lemmas.

Lemma 6 (see [11]). Let

$$
h(z)=1+\sum_{n=1}^{\infty} h_{n} z^{n}
$$

be analytic in $\mathbb{U}$ and

$$
k(z)=1+\sum_{n=1}^{\infty} k_{n} z^{n}
$$

be analytic and convex in $\mathbb{U}$. If $h(z) \prec k(z)$, then

$$
\left|h_{n}\right| \leq\left|k_{1}\right|, \quad(n \in N) .
$$


Lemma 7. Let $g(z) \in M S^{*}(1 / 2)$ and be given by (2). Then,

$$
\begin{gathered}
\mid b_{n} t^{n+1}+b_{1} b_{n-2} t^{n-1}+b_{2} b_{n-3} t^{n-2} \\
+\cdots+b_{n-3} b_{2} t^{3}+b_{1} b_{n-2} t^{2}+b_{n} \mid \\
\leq \frac{2}{n+1}, \quad(0<|t| \leq 1) .
\end{gathered}
$$

Proof. According to Corollary 3, we have $t z g(z) g(t z) \in$ $M S^{*}$, and if

$$
G(z)=\operatorname{tzg}(z) g(t z)=\frac{1}{z}+\sum_{n=1}^{\infty} c_{n} z^{n}
$$

then it is well known that

$$
\left|c_{n}\right| \leq \frac{2}{n+1} .
$$

Substituting the series expressions of $G(z)$ and $g(z)$ in (23) and comparing the coefficients of both sides of this equation, we get

$$
\begin{aligned}
c_{n}= & b_{n} t^{n+1}+b_{1} b_{n-2} t^{n-1}+b_{2} b_{n-3} t^{n-2} \\
& +\cdots+b_{n-3} b_{2} t^{3}+b_{1} b_{n-2} t^{2}+b_{n}
\end{aligned}
$$

On substituting the value from (25) in (24), we get the result.

Theorem 8. Let $-1 \leq B<A \leq 1$. If

$$
f(z)=\frac{1}{z}+\sum_{n=1}^{\infty} a_{n} z^{n} \in M K(t, A, B),
$$

then

$$
\begin{gathered}
\left|a_{1}\right| \leq 1 \\
\left|a_{n}\right| \leq \frac{A-B}{n}\left(1+\sum_{k=2}^{n} \frac{2}{k}\right)+\frac{2}{n(n+1)} \quad(n \in N \backslash\{1\}) .
\end{gathered}
$$

Proof. Suppose that $f \in M K(t, A, B)$. Then, we know that

$$
\frac{-z f^{\prime}(z)}{G(z)} \prec \frac{1+A z}{1+B z},
$$

where $G(z)=t z g(z) g(t z)$.

If we set

$$
q(z)=\frac{-z f^{\prime}(z)}{G(z)}
$$

it follows that

$$
q(z)=1+\sum_{n=1}^{\infty} d_{n} z^{n}
$$

In view of Lemma 6 , we know that

$$
\left|d_{n}\right| \leq A-B \quad(n \in N) .
$$

By substituting the series expression of functions $f, G$, and $q$ in (29), we obtain

$$
\begin{aligned}
& \left(1+d_{1} z+d_{2} z^{2}+\cdots+d_{n} z^{n}+\cdots\right) \\
& \quad \times\left(\frac{1}{z}+c_{1} z+c_{2} z^{2}+\cdots+c_{n} z^{n}+\cdots\right) \\
& =\frac{1}{z}-a_{1} z-2 a_{2} z^{2}-\cdots-n a_{n} z^{n}-\cdots .
\end{aligned}
$$

Since $f$ is univalent in $\mathbb{D}$, it is well known that

$$
\left|a_{1}\right| \leq 1 \text {. }
$$

On comparing the coefficient of $z^{n}$ in both sides of (32), we have

$$
\begin{aligned}
-n a_{n}= & c_{n}+d_{1} c_{n-1}+d_{2} c_{n-2} \\
& +\cdots+d_{n-1} c_{1}+d_{n+1} \quad(n \in N) .
\end{aligned}
$$

Substituting the values from (24) and (31) to (34), we have

$$
\begin{aligned}
n\left|a_{n}\right| \leq & (A-B)\left(1+\frac{2}{2}+\frac{2}{3}+\cdots+\frac{2}{n-1}+\frac{2}{n}\right) \\
& +\frac{2}{n+1} \\
\left|a_{n}\right| & \leq \frac{(A-B)}{n}\left(1+\sum_{k=2}^{n} \frac{2}{k}\right)+\frac{2}{n(n+1)} .
\end{aligned}
$$

This evidently completes the proof of Theorem 8 .

Theorem 9. Let $-1 \leq B<A \leq 1$. If

$$
g(z)=\frac{1}{z}+\sum_{n=1}^{\infty} b_{n} z^{n} \in M S^{*}\left(\frac{1}{2}\right)
$$

and $f \in \sum$ given by (1), satisfying the condition

$$
(1+|B|) \sum_{n=1}^{\infty} n\left|a_{n}\right|+(1+|A|) \sum_{n=1}^{\infty}\left|c_{n}\right| \leq(A-B),
$$

where $c_{n}$ is given by (25), then $f \in M K(t, A, B)$.

Proof. To prove $f \in M K(t, A, B)$, it suffices to show that

$$
\left|\frac{z f^{\prime}(z) / G(z)+1}{A+B\left(z f^{\prime}(z) / G(z)\right)}\right|<1,
$$

where $G(z)$ is given by (23). From (38), we know that

$$
\begin{aligned}
& (A-B)-|A| \sum_{n=1}^{\infty}\left|c_{n}\right|-|B| \sum_{n=1}^{\infty} n\left|a_{n}\right| \\
& \geq \sum_{n=1}^{\infty} n\left|a_{n}\right|+\sum_{n=1}^{\infty}\left|c_{n}\right| .
\end{aligned}
$$


Now, by maximum modulus principle and (40), we deduce that

$$
\begin{aligned}
& \left|\frac{z f^{\prime}(z) / G(z)+1}{A+B\left(z f^{\prime}(z) / G(z)\right)}\right| \\
& \quad=\left|\frac{\sum_{n=1}^{\infty} n a_{n} z^{n+1}+\sum_{n=1}^{\infty} c_{n} z^{n+1}}{(A-B)+A \sum_{n=1}^{\infty} c_{n} z^{n+1}+B \sum_{n=1}^{\infty} n a_{n} z^{n+1}}\right| \\
& \quad \leq \frac{\sum_{n=1}^{\infty} n\left|a_{n}\right|+\sum_{n=1}^{\infty}\left|c_{n}\right|}{|A-B|-|A| \sum_{n=1}^{\infty}\left|c_{n}\right|-|B| \sum_{n=1}^{\infty} n\left|a_{n}\right|} \leq 1 .
\end{aligned}
$$

This evidently completes the proof of Theorem 9.

Theorem 10. Let $-1 \leq B<A \leq 1$. If $f(z) \in M K(t, A, B)$, then

$$
\begin{aligned}
& f(z) *\left(\left(1+B e^{i \theta}\right) \frac{(2 z-1)}{z(1-z)^{2}}\right) \\
& +G(z) *\left(\left(1+A e^{i \theta}\right) \frac{\left(z^{2}-z+1\right)}{z(1-z)}\right) \neq 0, \\
& (z \in \mathbb{D}, 0<\theta<2 \pi) .
\end{aligned}
$$

Proof. Suppose that $f \in M K(t, A, B)$. Then, we know that

$$
\frac{-z f^{\prime}(z)}{G(z)} \neq \frac{1+A e^{i \theta}}{1+B e^{i \theta}} \quad(z \in \mathbb{D}, 0<\theta<2 \pi),
$$

where $G$ is given by (23). It is easy to see that the condition (43) can be written as

$$
\begin{array}{r}
z f^{\prime}(z)\left(1+B e^{i \theta}\right)+G(z)\left(1+A e^{i \theta}\right) \neq 0, \\
(z \in \mathbb{D}, 0<\theta<2 \pi) .
\end{array}
$$

Note that

$$
\begin{aligned}
& z f^{\prime}(z)=f(z) * \frac{2 z-1}{z(1-z)^{2}}, \\
& G(z)=G(z) * \frac{z^{2}-z+1}{z(1-z)} .
\end{aligned}
$$

By substituting (45) into (44), we get the desired assertion (42) of Theorem 10.

\section{Distortion Theorem}

Lemma 11 (see [12]). Let $\phi \in M S^{*}$. Then,

$$
\frac{(1-r)^{2}}{r} \leq|\phi(z)| \leq \frac{(1+r)^{2}}{r} \quad(|z|=r, 0<r<1) .
$$

Theorem 12. Let $-1 \leq B<A \leq 1$. If $f(z) \in M K(t, A, B)$, then

$$
\begin{array}{r}
\frac{(1-A r)(1-r)^{2}}{(1-B r) r^{2}} \leq\left|f^{\prime}(z)\right| \leq \frac{(1+A r)(1+r)^{2}}{(1+B r) r^{2}}, \\
(|z|=r ; 0<r<1) .
\end{array}
$$

Proof. Suppose that $f(z) \in M K(t, A, B)$. By definition, we have the transformation

$$
\frac{-z f^{\prime}(z)}{G(z)}=\frac{1+A w(z)}{1+B w(z)}, \quad w(z) \in \mathbb{U},
$$

which maps $|w(z)| \leq r$ in the closed disk

$$
\left|\frac{-z f^{\prime}(z)}{G(z)}-\frac{1-A B r^{2}}{1-B^{2} r^{2}}\right| \leq \frac{(A-B) r}{1-B^{2} r^{2}}, \quad|z|=r .
$$

This implies that

$$
\frac{1-A r}{1-B r} \leq\left|\frac{-z f^{\prime}(z)}{G(z)}\right| \leq \frac{1+A r}{1+B r}
$$

Thus, by virtue of Lemma 11, we readily get the desired result.

Remark 13. Taking $A=1, B=-1$, and $t=-1$, we obtain the results such as inclusion relation, coefficient inequality, convolution property, and distortion theorem derived by Wang et al. [1].

\section{Acknowledgment}

The authors would like to express their thanks to the referee for careful reading and suggestions made for the improvement of the paper.

\section{References}

[1] Z.-G. Wang, Y. Sun, and N. Xu, "Some properties of certain meromorphic close-to-convex functions," Applied Mathematics Letters, vol. 25, no. 3, pp. 454-460, 2012.

[2] C. Y. Gao and S. Q. Zhou, "On a class of analytic functions related to the starlike functions," Kyungpook Mathematical Journal, vol. 45, no. 1, pp. 123-130, 2005.

[3] Z.-G. Wang and D.-Z. Chen, "On a subclass of close-to-convex functions," Hacettepe Journal of Mathematics and Statistics, vol. 38, no. 2, pp. 95-101, 2009.

[4] Z.-G. Wang, C.-Y. Gao, and S.-M. Yuan, "On certain new subclass of close-to-convex functions," Matematichki Vesnik, vol. 58, no. 3-4, pp. 119-124, 2006.

[5] J. Kowalczyk and E. Leś-Bomba, "On a subclass of close-toconvex functions," Applied Mathematics Letters, vol. 23, no. 10, pp. 1147-1151, 2010.

[6] Q.-H. Xu, H. M. Srivastava, and Z. Li, "A certain subclass of analytic and close-to-convex functions," Applied Mathematics Letters, vol. 24, no. 3, pp. 396-401, 2011.

[7] B. Şeker, "On certain new subclass of close-to-convex functions," Applied Mathematics and Computation, vol. 218, no. 3, pp. 1041-1045, 2011.

[8] N. E. Cho, O. S. Kown, and V. Ravichandran, "Coefficient, distortion and growth inequalities for certain close-to-convex functions," Journal of Inequalities and Applications, vol. 201, article 100, 2011.

[9] Y. J. Sim and O. S. Kown, "A subclass of meromorphic closeto-convex function of Janowski's type," International Journal of Mathematics and Mathematical Sciences, vol. 2012, Article ID 682162, 12 pages, 2012. 
[10] M. S. Liu, "On a subclass of $p$-valent close-to-convex functions of type $\alpha$ and order $\beta$," Journal of Mathematical Study, vol. 30, no. 1, pp. 102-104, 1997 (Chinese).

[11] W. Rogosinski, "On the coefficients of subordinate functions," Proceedings of the London Mathematical Society Series 2, vol. 48, pp. 48-82, 1943.

[12] Ch. Pommerenke, “On meromorphic starlike functions," Pacific Journal of Mathematics, vol. 13, pp. 221-235, 1963. 


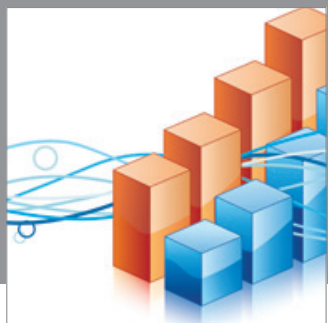

Advances in

Operations Research

mansans

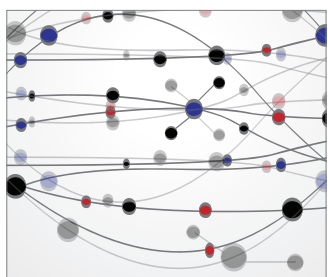

The Scientific World Journal
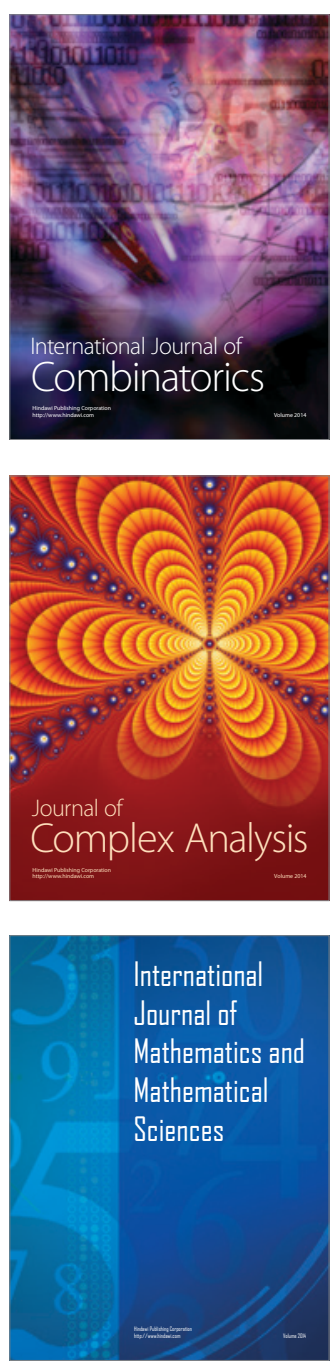
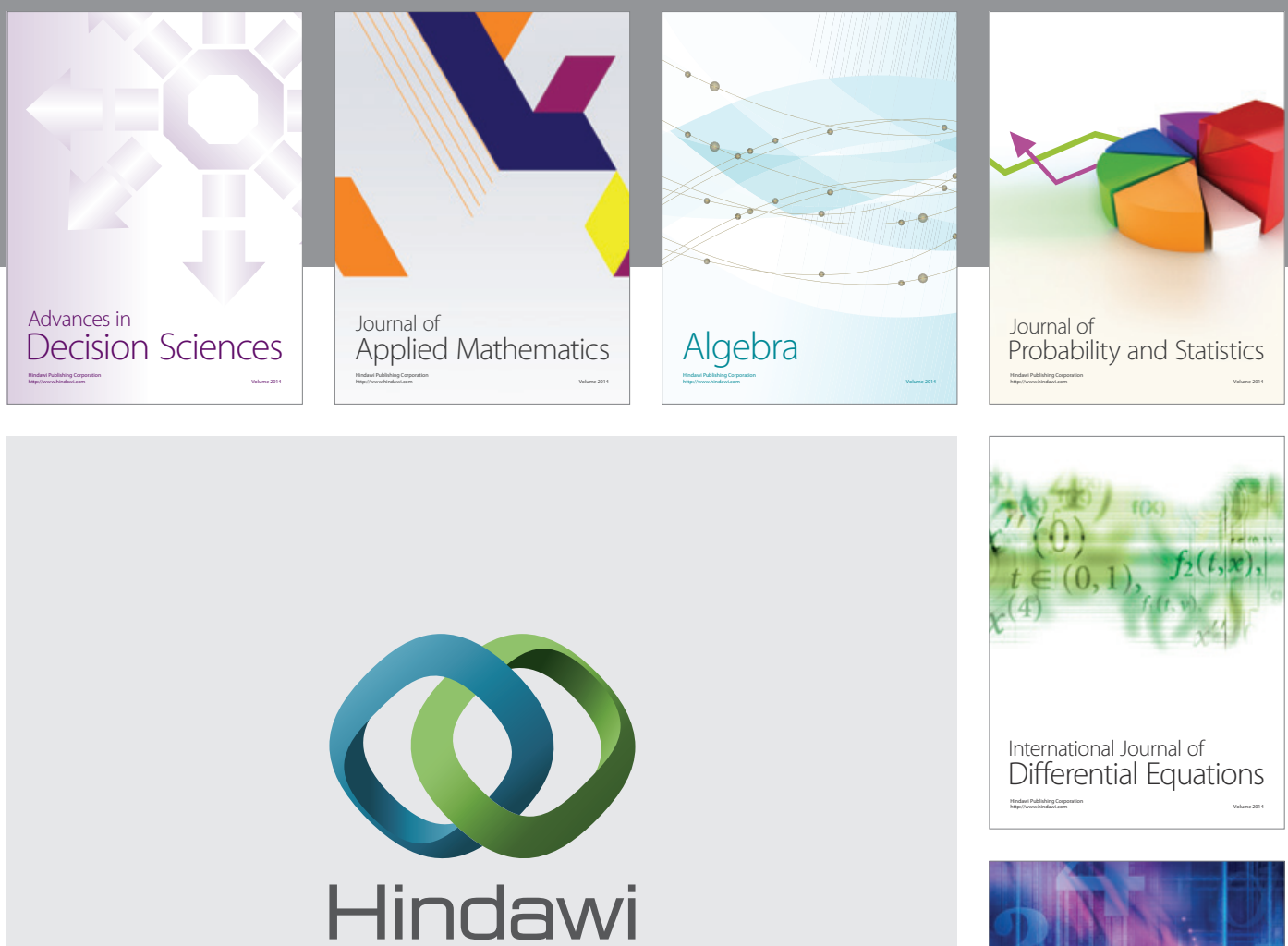

Submit your manuscripts at http://www.hindawi.com
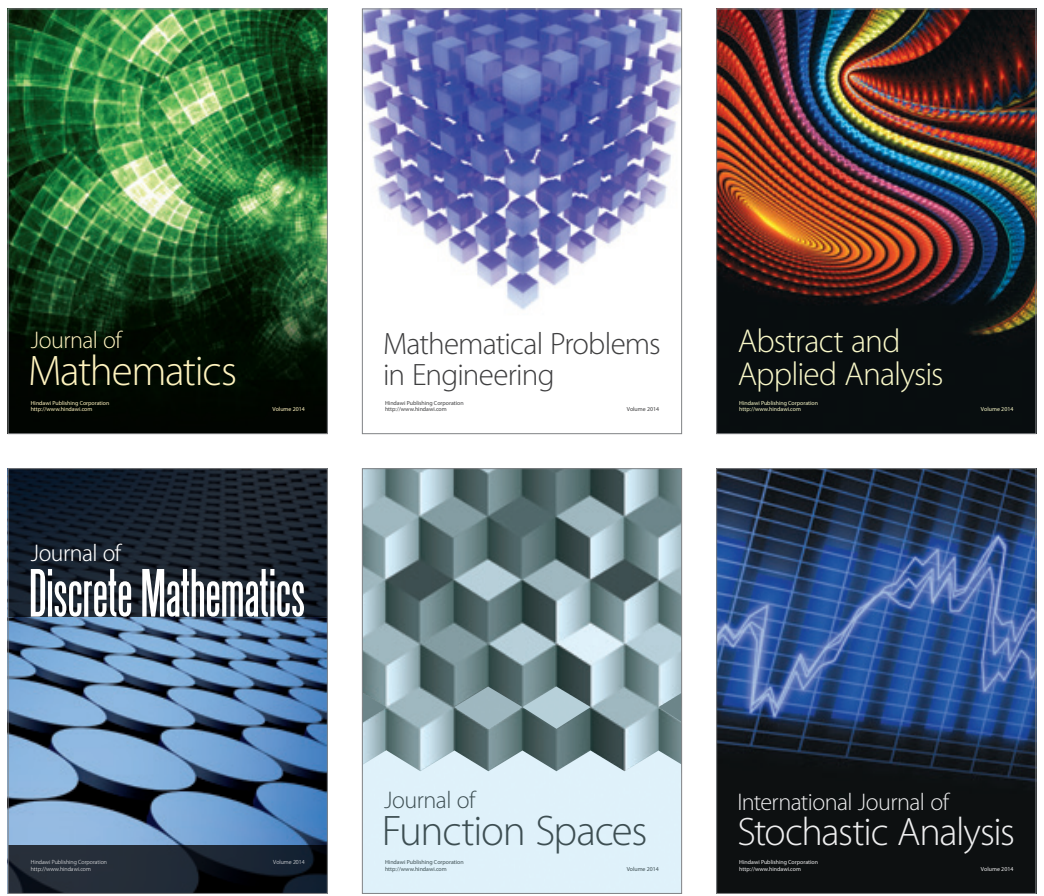

Journal of

Function Spaces

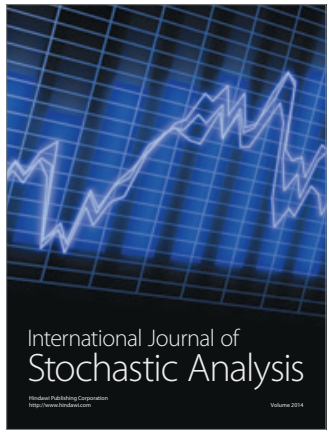

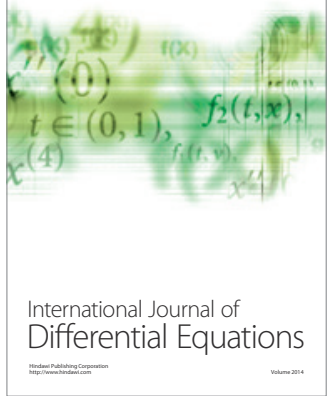
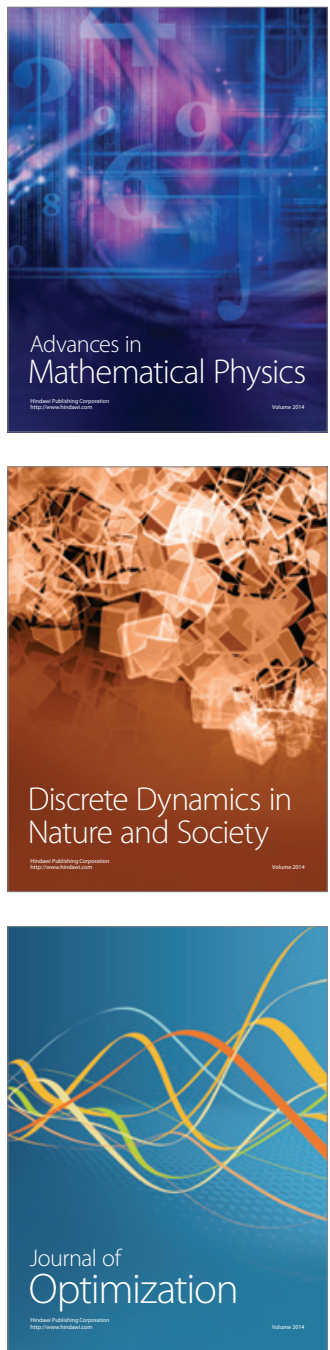